\title{
Tumores metastáticos na região bucomaxilofacial: uma revisão sistemática
}

\author{
Metastatic tumors in the maxillofacial region: a systematic review \\ Tumores metastásicos en la región maxilofacial: una revisión sistemática
}

Nívia Castro Binda

ORCID: https://orcid.org/0000-0002-0409-4265

Universidade Federal do Espírito Santo, Brasil E-mail: nivia_sgp@hotmail.com

Ana Luiza Castro Binda

ORCID: https://orcid.org/0000-0002-6664-2538

Escola São Francisco de Assis, Brasil

E-mail: aninha_sgp@hotmail.com

Rodolfo Alves de Pinho

ORCID: https://orcid.org/0000-0002-2151-6642

Faculdade Vértice, Brasil

E-mail: rodolfoalvesmtp@gmail.com

Matheus Almeida Ramalho

ORCID: https://orcid.org/0000-0002-5607-1313

Universidade Federal de Jataí, Brasil

E-mail: matheusramalho@discente.ufg.br

Bruna Peixoto Girard

ORCID: https://orcid.org/0000-0003-0233-666X

Centro Universitário Cesmac, Brasil

E-mail: brunapeixoto5@hotmail.com

Nívia Delamoniky Lima Fernandes

ORCID: https://orcid.org/0000-0001-8439-2117

Centro Universitário Inta, Brasil

E-mail: delamonikynivia@gmail.com

Jefferson Douglas Lima Fernandes

ORCID: https://orcid.org/0000-0002-5231-3813

Federal University of Ceará, Brasil

E-mail: jefferson.odonto97@gmail.com

Marcieli Borba do Nascimento ORCID: https://orcid.org/0000-0002-8087-0801 Universidade Estadual do Centro-Oeste, Brasil E-mail: marcieli_bn@hotmail.com

Ana Cristina Freitas da Silva

ORCID: https://orcid.org/0000-0001-5026-2250

Universidade do Extremo Sul Catarinense, Brasil E-mail: Anacff@unesc.net

José Victor Lima Silva

ORCID: https://orcid.org/0000-0002-8539-5456

Centro Universitário Christus, Brasil

E-mail: victorlimasv@ hotmail.com

Vittor Dorinato de Santana Sátiro ORCID: https://orcid.org/0000-0002-7069-0807 Universidade Paulista, Brasil

E-mail: vittorodonto@gmail.com

Maria Karoline Gomes da Silva ORCID: https://orcid.org/0000-0001-8786-3332

Centro Universitário Cesmac, Brasil

E-mail: karolinegomes201@gmail.com

Bruno Mariano Ribeiro Braga ORCID: https://orcid.org/0000-0003-4312-8170

Universidade de São Paulo, Brasil E-mail: brunomrbraga@usp.br

Gisele de Oliveira Leandro Moro ORCID: https://orcid.org/0000-0002-6883-3565 Universidade do Extremo Sul Catarinense, Brasil E-mail: giedionei@unesc.net

Zildenilson da Silva Sousa

ORCID: https://orcid.org/0000-0002-2707-6123 Centro Universitário Maurício de Nassau, Brasil

E-mail: zildenilsonsilva@gmail.com 


\author{
Jaqueline Lopes Reis \\ ORCID: https://orcid.org/ 0000-0003-1685-0336 \\ Universidade de Rio Verde, Brasil \\ E-mail: jaquelreis@ hotmail.com \\ Myra Jurema da Rocha Leão \\ ORCID: https://orcid.org/0000-0003-1782-5239 \\ Santa Casa de Misericórdia de Maceió, Brasil \\ E-mail: myra.jurema.rocha.leao@gmail.com
}

\begin{abstract}
Resumo
A metástase é consequência de uma cascata biológica complexa, que se inicia com o desprendimento de células tumorais de um sítio primário e, por via hematogênica ou linfática, se disseminam para outras regiões, onde passam a residir e se multiplicar, formando os chamados "implantes secundários" nesses novos locais. As lesões metastáticas na região bucomaxilofacial representam cerca de $1 \%$ de todos os tumores malignos desta região, sendo a cavidade oral o principal sítio primário dessas neoplasias. O câncer da região bucomaxilofacial apresenta altas taxas de morbimortalidade. Assim, é necessário que o médico e/ou cirurgião dentista leve em consideração as metástases para essa região, pois, apesar de ser um caso raro, indicam que a neoplasia já se encontra em estágio avançado. Diante do exposto, este estudo teve como objetivo realizar uma revisão sistemática da literatura sobre os locais do tumor primário que possuem metástase para a região bucomaxilofacial, bem como o local da metástase, gênero mais acometido e o tipo histológico mais comum. Para a construção deste artigo foi feito um levantamento bibliográfico nas bases de dados SciVerse Scopus, Scientific Eletronic Library Online (Scielo), U.S. National Library of Medicine (PUBMED) e ScienceDirect, com auxílio do Mendeley. Os artigos foram coletados no período de abril a agosto de 2021 e contemplados entre os anos de 2010 a 2021. A partir de uma revisão sistemática da literatura, observou-se 282 casos de metástase para a região bucomaxilofacial. Os sítios primários que mais metastatizaram foram pulmão, rim, mama e fígado. O gênero mais acometido foi o masculino e a mandíbula foi o local mais acometido, seguido por gengiva e língua. O tipo histológico mais comum foi o carcinoma.
\end{abstract}

Palavras-chave: Metástase; Região oral; Mandíbula; Incidência.

\begin{abstract}
Metastasis is a consequence of a complex biological cascade, which begins with the detachment of tumor cells from the primary site and, via blood or lymph, spread to other sites, where they form a new neoplastic colony. They represent about $1 \%$ of all malignant tumors in this region, the oral cavity being the site most affected by neoplasms. Oral and maxillofacial cancer has high morbidity and mortality rates. Thus, it is necessary that the physician and/or dental surgeon take into account metastases to this region, as, despite being a rare case, they are signs of disseminated neoplasms. Given the above, this study aimed to conduct a systematic review of the literature on the sites of the primary tumor that have metastases to the maxillofacial region, as well as the site of metastasis, the most affected gender and the most common histological type. For the construction of this article, a bibliographic survey was carried out in the SciVerse Scopus, Scientific Electronic Library Online (Scielo), U.S. National Library of Medicine (PUBMED) and ScienceDirect databases, with the help of Mendeley. The articles were collected from April to August 2021 and covered between 2010 and 2021. From a systematic review of the literature, 282 cases of metastasis to the maxillofacial region were observed. The primary sites that metastasize the most are lung, kidney, breast and liver. The most affected gender is the male and the mandible is the most affected location, followed by the gums and tongue. The most common histological type is carcinoma.
\end{abstract}

Keywords: Metastasis; Oral region; Jaw; Incidence.

\title{
Resumen
}

La metástasis es consecuencia de una cascada biológica compleja, que comienza con el desprendimiento de las células tumorales del sitio primario y, a través de la sangre o la linfa, se disemina a otros sitios, donde forman una nueva colonia neoplásica. Representan alrededor del $1 \%$ de todos los tumores malignos de esta región, siendo la cavidad bucal el sitio más afectado por las neoplasias. El cáncer oral y maxilofacial tiene altas tasas de morbilidad y mortalidad. Por tanto, es necesario que el médico y / o dentista tenga en cuenta las metástasis a esta región, ya que, a pesar de ser un caso raro, son signos de neoplasias diseminadas. Dado lo anterior, este estudio tuvo como objetivo realizar una revisión sistemática de la literatura sobre los sitios del tumor primario que presentan metástasis a la región maxilofacial, así como el sitio de metástasis, el género más afectado y el tipo histológico más común. Para la construcción de este artículo se realizó un relevamiento bibliográfico en las bases de datos SciVerse Scopus, Scientific Electronic Library Online (Scielo), U.S. National Library of Medicine (PUBMED) y ScienceDirect, con la ayuda de Mendeley. Los artículos fueron recolectados de abril a agosto de 2021 y cubiertos entre 2010 y 2021. A partir de una revisión sistemática de la literatura, se observaron 282 casos de metástasis a la región maxilofacial. Los sitios primarios que más metastatizan son pulmón, riñón, mama e hígado. El género más afectado es el masculino y la mandíbula es la ubicación más afectada, seguida de las encías y la lengua. El tipo histológico más común es el carcinoma.

Palabras clave: Metástasis; Región oral; Mandíbula; Incidencia. 


\section{Introdução}

O câncer é um termo que se refere ao crescimento incomum e desordenado de células que podem invadir tecidos adjacentes ou órgãos à distância (Canto 2017). O processo de formação é chamado de carcinogênese ou oncogênese e, se inicia pela consequência de efeitos cumulativos de diferentes agentes carcinógenos, que são causadores do início, promoção, promoção e inibição do tumor (Lawes, Danford \& Di Palma 2013).

Os fatores de risco associados a essa malignidade envolvem o consumo excessivo de álcool e/ou tabagismo, alimentação, infecção pelo papilomavirus humano (HPV), exposição à radiação solar sem proteção, sobrepeso corporal e herança genética (Reymond, d’Água, \& Ridley 2013; Zalaudek et al. 2015). Em homens, de acordo com o Instituto Nacional do Câncer (INCA), o tipo de câncer mais prevalente é o de próstata, seguido por cólon, reto, traqueia, brônquios e pulmões. Já em mulheres, a mama é o local mais acometido por neoplasias, seguido pelo cólon, reto e colo do útero.

A metástase é consequência de uma cascata biológica complexa, que se inicia com o desprendimento das células tumorais do sítio primário e, por via hematogênica ou linfática, se disseminam para outras regiões, onde passam a residir e se multiplicar, formando os chamados “implantes secundários" nesses novos sítios (Nawale et al. 2016). As metástases representam cerca de $1 \%$ de todos os tumores malignos da região, sendo a cavidade oral o local mais acometido pelas neoplasias (Metzger et al. 2021).

O câncer da região bucomaxilofacial apresenta altas taxas de morbimortalidade (Liu, Vargo, \& Bilodeau 2018). Assim, é necessário que o médico e/ou cirurgião dentista leve em consideração as metástases para essa região, pois, apesar de ser um caso raro, são sinais de doença disseminadas (Irani 2016).

Diante do exposto, este estudo teve como objetivo realizar uma revisão sistemática da literatura sobre os locais do tumor primário que possuem metástase para a região bucomaxilofacial, bem como o local da metástase, gênero mais acometido e o tipo histológico mais comum.

\section{Metodologia}

Trata-se de uma revisão sistemática de literatura. Essa metodologia permite incorporar um espectro maior de resultados relevantes, por meio de um resumo de todos estudos sobre determinado tema, nesse caso, metástases na região bucomaxilofacial (Waltz 2013).

A fim de que haja direcionamento na pesquisa, delineou-se como questão norteadora: "quais os tumores primários possuem metástase na região bucomaxilofacial e qual o seu tipo histológico?”, “qual a área anatômica da região bucomaxilofacial mais acometida pelas metástases tumorais?" e "qual a idade e gênero mais acometido pela metástase tumoral na região bucomaxilofacial?".

\subsection{Busca Estratégica}

Para a construção deste artigo foi feito um levantamento bibliográfico nas bases de dados SciVerse Scopus, Scientific Eletronic Library Online (Scielo), U.S. National Library of Medicine (PUBMED) e ScienceDirect, com auxílio do Mendeley. Os artigos foram coletados no período de janeiro a agosto de 2021 e contemplados entre os anos de 2010 a 2021.

A estratégia de pesquisa desenvolvida para identificar os artigos incluídos e avaliados para este estudo baseou-se nos descritores contidos na lista dos Descritores em Ciência da Saúde (DeCS) e suas combinações no idioma português e inglês: [(cancer OR câncer OR neoplasma OR neoplasm OR tumor) AND (boca OR mouth OR oral OR mandíbula OR mandible OR jaw OR maxila OR maxilar OR maxilla OR língua OR tongue OR gengiva OR gingiva) AND (metastase OR metastasis)] 


\subsection{Critérios de inclusão}

Considerou-se como critério de inclusão os artigos completos disponíveis na íntegra nas bases de dados citadas, nos idiomas inglês e português e relacionados com o objetivo deste estudo.

\subsection{Critérios de exclusão}

Os critérios de exclusão foram artigos incompletos, duplicados, resenhas, estudos in vitro, resumos e artigos sobre tumores primários na região bucomaxilofacial.

\subsection{Seleção de estudos}

A estratégia de pesquisa baseou-se na leitura dos títulos para encontrar estudos que investigassem a temática da pesquisa. Caso atingisse esse primeiro objetivo, posteriormente, os resumos eram lidos e, persistindo na inclusão, era feita a leitura do artigo completo. Quando havia dúvida sobre a inclusão, o artigo era lido por outro autor e, a decisão de inclusão ou exclusão era tomada em consenso.

\subsection{Coleta de dados}

$\mathrm{Na}$ sequência metodológica foi realizada a busca e leitura na íntegra dos artigos pré-selecionados, os quais foram analisados para inclusão da amostra. Cada pesquisador preencheu um formulário padronizado no Microsoft Excel (2010) para tabular os seguintes dados extraídos dos artigos: autores, ano de publicação, faixa etária, gênero, número de casos, local do tumor primário, local de metástase na região bucomaxilofacial e tipo histológico tumoral.

\section{Resultados e Discussão}

Com base na revisão de literatura feita nas bases de dados eletrônicas citadas, foram identificados 3159 artigos científicos, dos quais 468 estavam duplicados com dois ou mais índices. Após a leitura e análise do título e resumos dos demais artigos outros 2611 foram excluídos. Assim, 80 artigos foram lidos na integra e, com base nos critérios de inclusão e exclusão, apenas 12 artigos foram selecionados para compor este estudo. O fluxograma com detalhamento de todas as etapas de seleção está na Figura 1. 
Figura 1 - Fluxograma de identificação e seleção dos estudos.

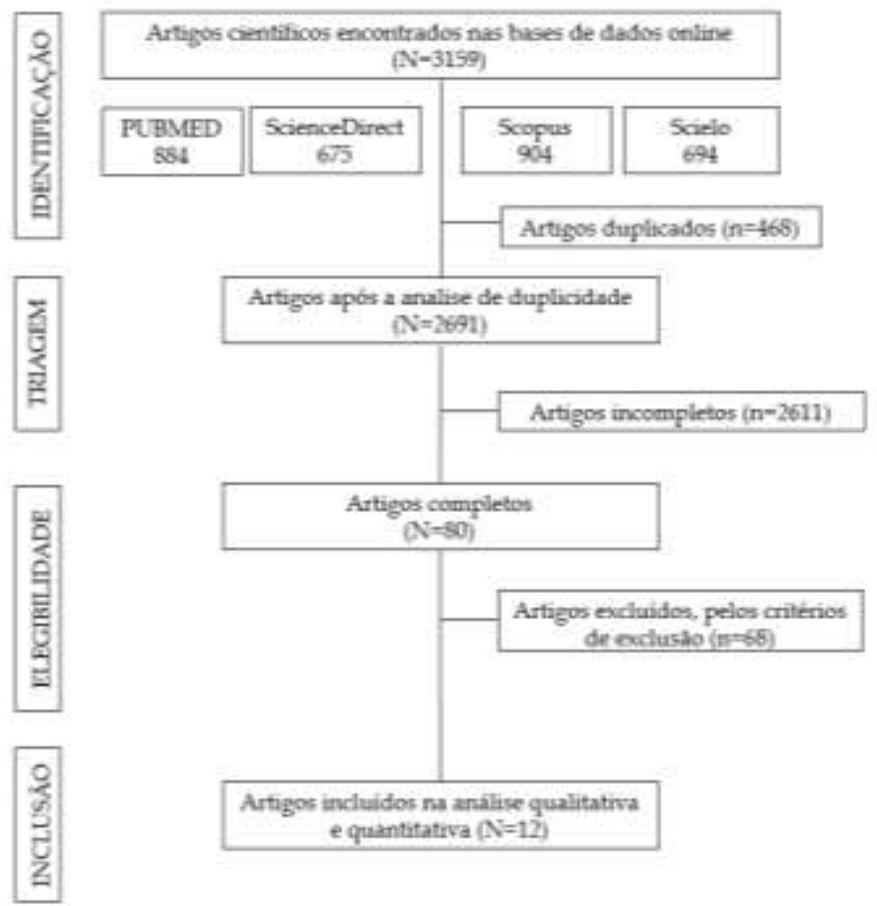

Fonte: Autores (2021).

A partir a revisão sistemática da literatura, observou-se 282 casos de metástase para a região bucomaxilofacial. A Tabela 1 relata as características dos 12 artigos incluídos no trabalho.

Tabela 1 - Características descritivas dos estudos incluídos $(\mathrm{N}=12)$.

\begin{tabular}{|c|c|c|c|c|c|c|}
\hline $\begin{array}{l}\text { Autor, } \\
\text { Ano }\end{array}$ & $\begin{array}{l}\text { Número } \\
\text { de casos }\end{array}$ & Gênero & Faixa etária & $\begin{array}{c}\text { Local do } \\
\text { Tumor primário }\end{array}$ & Local da metástase & Tipo Histológico \\
\hline \multirow[t]{5}{*}{$\begin{array}{l}\text { Chen et al. } \\
2020\end{array}$} & 13 & M: 11 & $39-78$ & Fígado (9) & Gengiva (8) & Carcinoma (11) \\
\hline & & F: 2 & & Pâncreas (1) & Mucosa Alveolar (2) & Adenocarcinoma (2) \\
\hline & & & & Cólon (1) & Mucosa Bucal (2) & \\
\hline & & & & Tireóide (1) & Mucosa palatina (1) & \\
\hline & & & & $\operatorname{Rim}(1)$ & & \\
\hline \multirow[t]{12}{*}{$\begin{array}{l}\text { Owosho et } \\
\text { al. } 2016\end{array}$} & 44 & M: 24 & 3 a 85 & Pulmão (10) & Mandíbula (20) & Carcinomas (18) \\
\hline & & F: 20 & & $\operatorname{Rim}(7)$ & Gengiva (11) & Adenocarcioma (17) \\
\hline & & & & Mama (5) & Mucosa Bucal (5) & Neuroblastoma (3) \\
\hline & & & & Glândula Adrenal (4) & Mucosa Palatina (3) & Sarcoma (3) \\
\hline & & & & Cólon (4) & Língua (3) & $\begin{array}{l}\text { Tumor de Células } \\
\text { Germinativas (1) }\end{array}$ \\
\hline & & & & Próstata (2) & Maxila (2) & Mesotelioma (1) \\
\hline & & & & Fígado (1) & & Retinobastoma (1) \\
\hline & & & & Pancreaticobiliar (1) & & \\
\hline & & & & Ureter (1) & & \\
\hline & & & & Útero (1) & & \\
\hline & & & & Tireoide (1) & & \\
\hline & & & & Nádega (1) & & \\
\hline
\end{tabular}




\begin{tabular}{|c|c|c|c|c|c|c|}
\hline & & & & $\begin{array}{l}\text { Testículo (1) } \\
\text { Estômago (1) } \\
\text { Mediastino (1) } \\
\text { Desconhecido (2) }\end{array}$ & & \\
\hline \multirow[t]{6}{*}{$\begin{array}{l}\text { Shimono et } \\
\text { al. } 2021\end{array}$} & 14 & H: 11 & $48-89$ & Pulmão (7) & Mandíbula (7) & Adenocarcinoma (7) \\
\hline & & M: 3 & & $\operatorname{Rim}(3)$ & Língua (4) & Carcinoma (8) \\
\hline & & & & Pélvis Renal (1) & Gengiva (2) & \\
\hline & & & & Estomago (1) & Maxila (1) & \\
\hline & & & & Tireoide (1) & & \\
\hline & & & & Bexiga (1) & & \\
\hline \multirow[t]{12}{*}{$\begin{array}{l}\text { Kaplan et } \\
\text { al. } 2019\end{array}$} & 60 & H: 25 & $17-87$ & Rins (12) & Mandíbula (26) & $\mathrm{NC}$ \\
\hline & & F: 35 & & Mama (9) & Gengiva (19) & \\
\hline & & & & Pele (8) & Mucosa Bucal (5) & \\
\hline & & & & Pulmão (7) & Maxila (5) & \\
\hline & & & & Tecido mole (5) & Vestíbulo (2) & \\
\hline & & & & Cólon (4) & Assolho bucal (1) & \\
\hline & & & & $\begin{array}{ll}\text { Trato } & \text { aerodigestivo } \\
\text { superior (4) }\end{array}$ & Lábio (1) & \\
\hline & & & & Tireóide (2) & Língua (1) & \\
\hline & & & & Próstata (2) & & \\
\hline & & & & Osso (1) & & \\
\hline & & & & Fígado (1) & & \\
\hline & & & & Desconhecido (5) & & \\
\hline \multirow{5}{*}{$\begin{array}{l}\text { Andabak } \\
\text { Rogulj et } \\
\text { al., } 2018\end{array}$} & 10 & M: 4 & $51-84$ & Rins (5) & Mandíbula (7) & Adenocarcinoma (6) \\
\hline & & F: 6 & & Pulmão (2) & Maxila (3) & Carcinoma (2) \\
\hline & & & & Mama (1) & & $\begin{array}{l}\text { Câncer de pulmão celular } \\
\text { (1) }\end{array}$ \\
\hline & & & & Cólon (1) & & Mama tubular invasiva (1) \\
\hline & & & & Desconhecido (1) & & \\
\hline \multirow[t]{5}{*}{$\begin{array}{l}\text { Maschino } \\
\text { et al. } 2013\end{array}$} & 23 & M: 17 & Média 64 & Pulmão (9) & $\begin{array}{l}\text { Gengiva e mucosa } \\
\text { alveolar (16) }\end{array}$ & Carcinoma (12) \\
\hline & & F: 6 & & Tecido mole (6) & Língua (7) & Adenocarcinoma (7) \\
\hline & & & & Rins (4) & & Sarcoma (4) \\
\hline & & & & Mama (2) & & \\
\hline & & & & Tireóide (2) & & \\
\hline \multirow[t]{7}{*}{$\begin{array}{l}\text { Lee and } \\
\text { Lee } 2017\end{array}$} & 21 & M: 13 & $31-83$ & Pulmão (7) & Mandíbula (15) & Carcinoma (15) \\
\hline & & F: 8 & & Fígado (5) & Mandíbula (6) & Adenocarcinoma (6) \\
\hline & & & & Mama (4) & Orofaringe (1) & \\
\hline & & & & Tireóide (2) & Glândula Parótida (1) & \\
\hline & & & & Reto (1) & Língua (1) & \\
\hline & & & & $\operatorname{Rim}(1)$ & Orofaringe (1) & \\
\hline & & & & Testículos (1) & & \\
\hline
\end{tabular}


2016

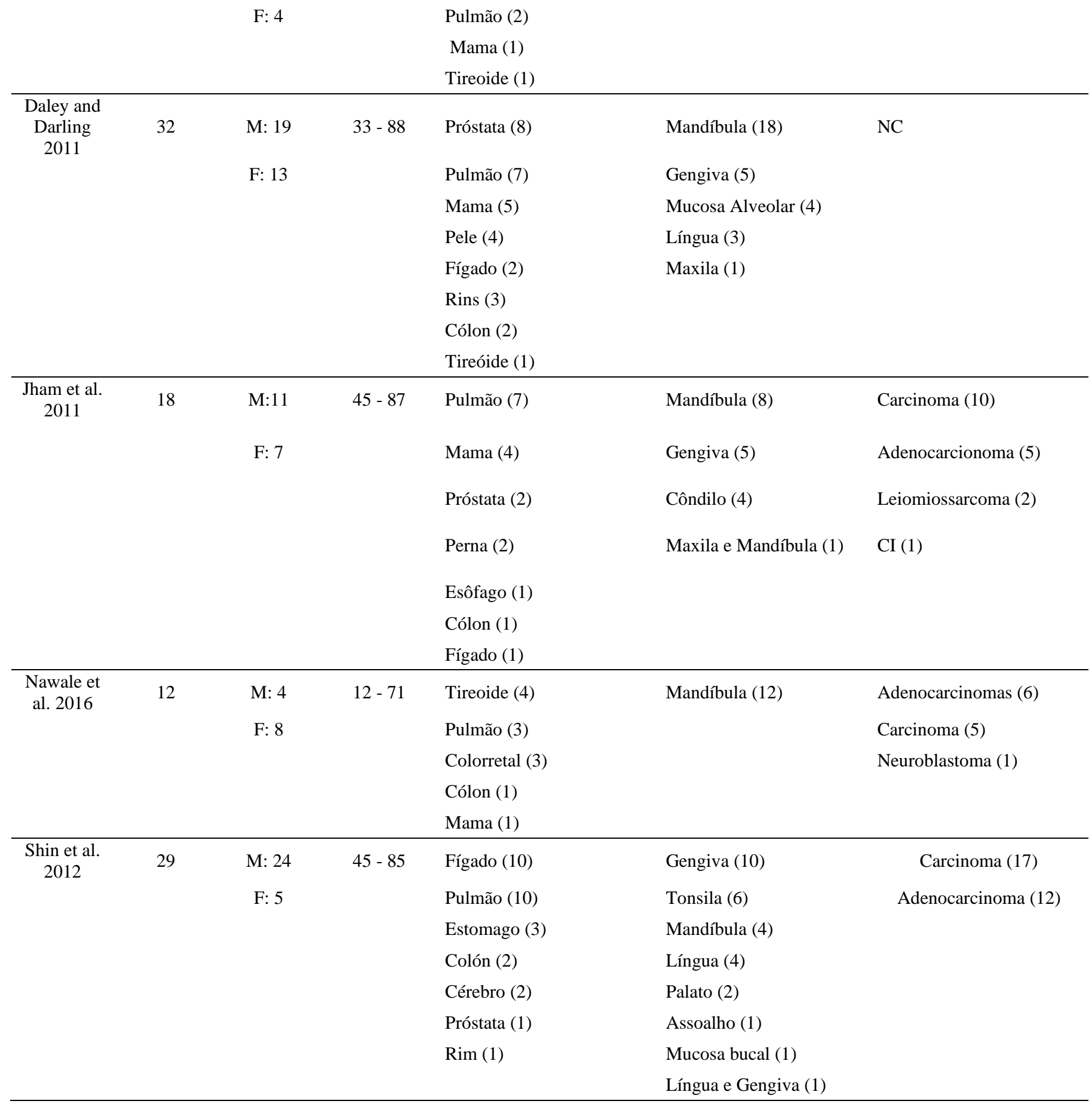

M: sexo masculino; F: sexo feminino; NC: não consta; CI: câncer indiferenciado. Fonte: Autores.

A fim de melhor compreensão dos dados, os resultados foram dispostos de acordo com o local do tumor primário (Figura 2), gênero dos pacientes acometidos (Figura 3), local da metástase (Figura 4) e tipo histológico (Figura 5).

O pulmão mostrou-se como o local de maior recorrência de metástase para a região bucomaxilofacial, representando cerca de $25 \%$ de todos os outros tumores analisados. Além disso, foi o sítio primário mais ocorrente em seis dos doze artigos incluídos nessa revisão sistemática (Andabak Rogulj et al. 2018; Jham et al. 2011; Maschino et al. 2013; Owosho et al. 2016; Shimono et al. 2021; Shin et al. 2012). Seguido do pulmão, o rim apresentou-se com recorrência de 13\%, mama com 11\% e fígado com $10 \%$ (Figura 2). 
Figura 2 - Local do tumor primário.

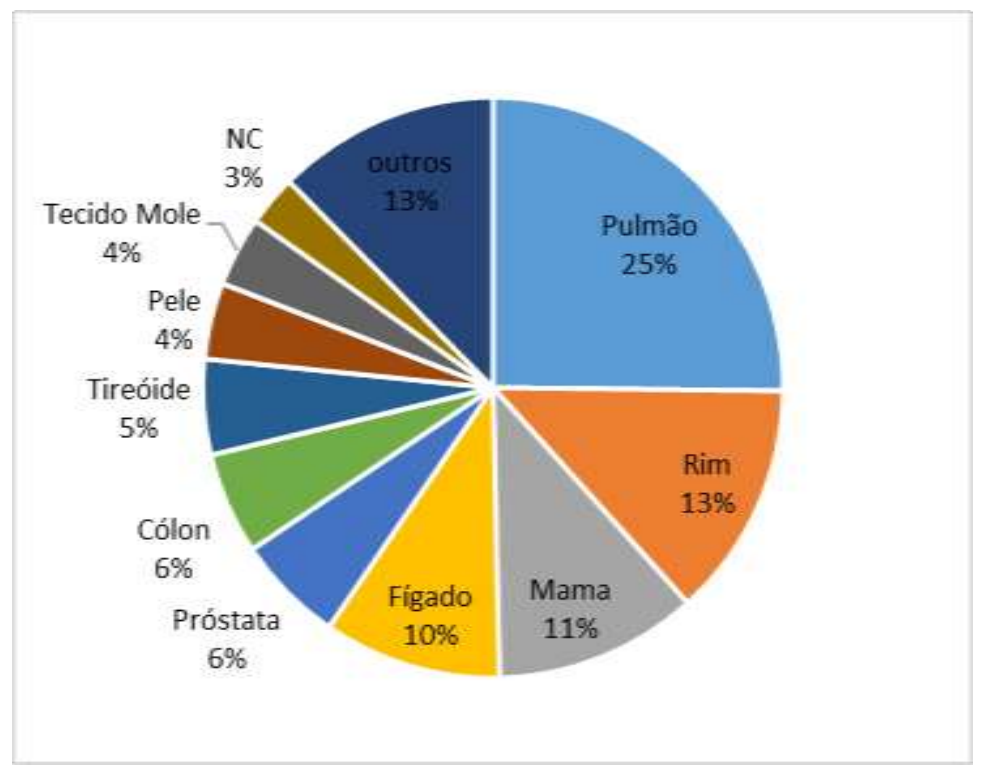

Fonte: Autores (2021).

O câncer afeta, principalmente homens, com idade superior a 40 anos. Isso ocorre, pois, o sexo masculino é mais exposto aos fatores de risco, como tabaco e etilismo. O presente estudo corrobora com tal afirmação, visto que a proporção ente homens e mulheres acometidos por metástase na região bucomaxilofacial foi de 1,41:1 (Figura 3).

Figura 3 - Relação entre homens e mulheres.

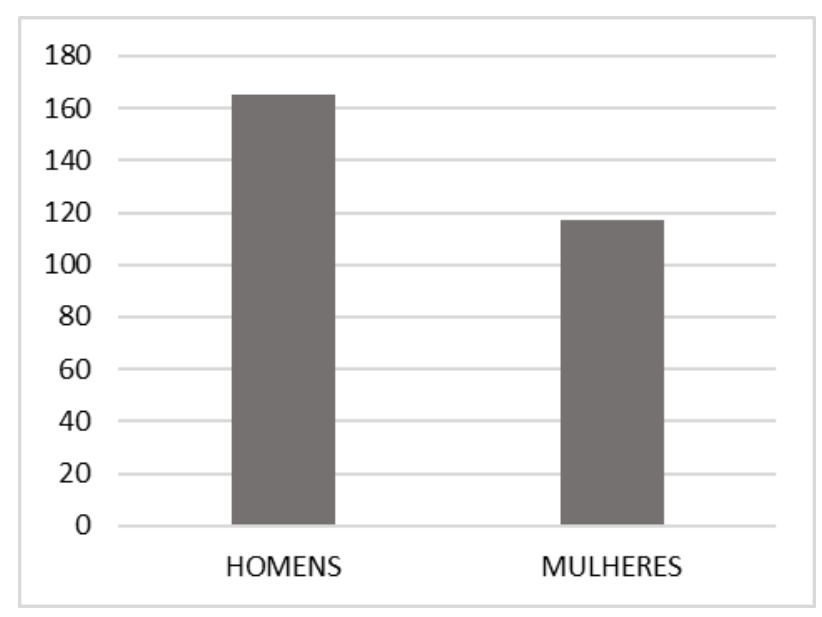

Fonte: Autores (2021).

Neste estudo, a faixa etária variou entre 3 a 88 anos. Sabe-se que o risco de um indivíduo desenvolver câncer aumenta gradativamente a medida que ele envelhece. No entanto, embora seja a minoria dos casos, houve relato de crianças, com idade inferior a 18 anos, acometidas por metástase na região bucomaxilofacial (Nawale et al. 2016; Owosho et al. 2016).

Considerando o local anatômico da região bucomaxilofacial, a mandíbula apresentou-se com maior incidência, representando um total de 44\% dos casos (Figura 4) (Andabak Rogulj et al. 2018; Cai et al. 2016; Daley \& Darling 2011; Jham et al. 2011; Kaplan et al. 2019; Lee \& Lee 2017; Maschino et al. 2013; Nawale et al. 2016; Owosho et al. 2016 ; Shimono et al. 2021). Nesses estudos Jham et al. 2011, Lee and Lee 2017 Owosho et al. 2016 e Shimono et al. 2021 relataram que câncer 
primário no pulmão é que mais acomete a mandíbula. Andabak Rogulj et al. 2018 e Kaplan et al. 2019 descreveram a predileção do tumor de rim como origem de metástase da mandíbula. Daley and Darling 2011 descreveram o tumor de próstata como sítio primário que mais acomete a mandíbula. Nawale et al. 2016 apontou o câncer de tireóide é o que mais causa metástase para a mandíbula e no estudo feito por Cai et al. 2016 houve similaridade entre os números de câncer de próstata e de pulmão que ocasionaram metástase para a mandíbula.

Figura 4 - Locais de metástase na região bucomaxilofacial.

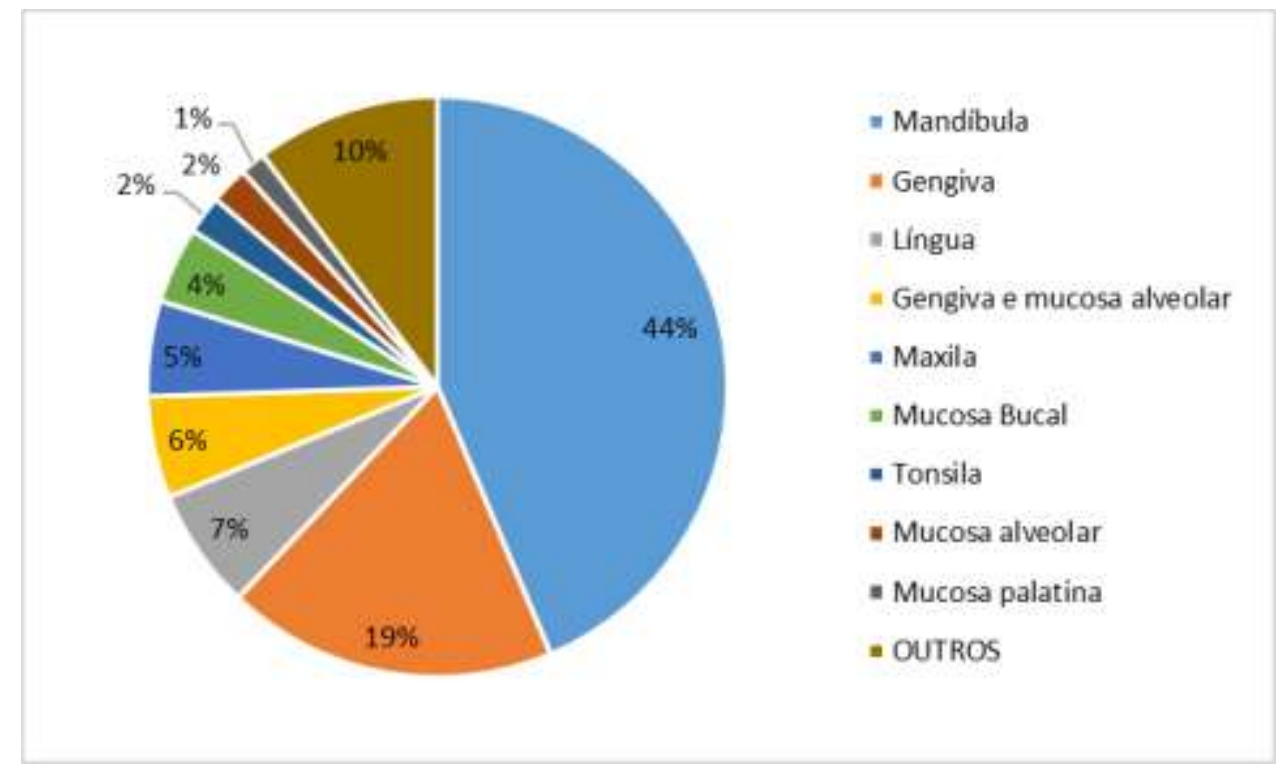

Fonte: Autores (2021).

Em relação a metástase para os tecidos moles da região bucomaxilofacial, a gengiva foi a mais acometida, representando cerca de $19 \%$ dos casos. Os estudos realizados por Chen et al. 2020, Maschino et al. 2013 e Shin et al. 2012 mostraram que, dentre os sítios de metástase da região bucomaxilofacial, a gengiva foi o local mais recorrente. Destes estudos, Chen et al. 2020 citaram o câncer de pulmão como sítio primário para essas metástases. Já Maschino et al. apresentaram o cancer de fígado como tumor primário e Shin et al. 2012 retrataram os mesmos valores de ocorrência de metástase na gengiva provenientes do câncer de pulmão ou fígado.

Dentre os 12 artigos selecionados, apenas 10 contemplavam o tipo histológico tumoral. Destes, observou-se que o tipo histológico mais acometido por metástase foi, na sua maioria, o carcinoma com $49 \%$, adenocarcinoma com $26 \%$ e sarcoma com 9\% (Figura 5). 
Figura 5 - Tipo histológico.

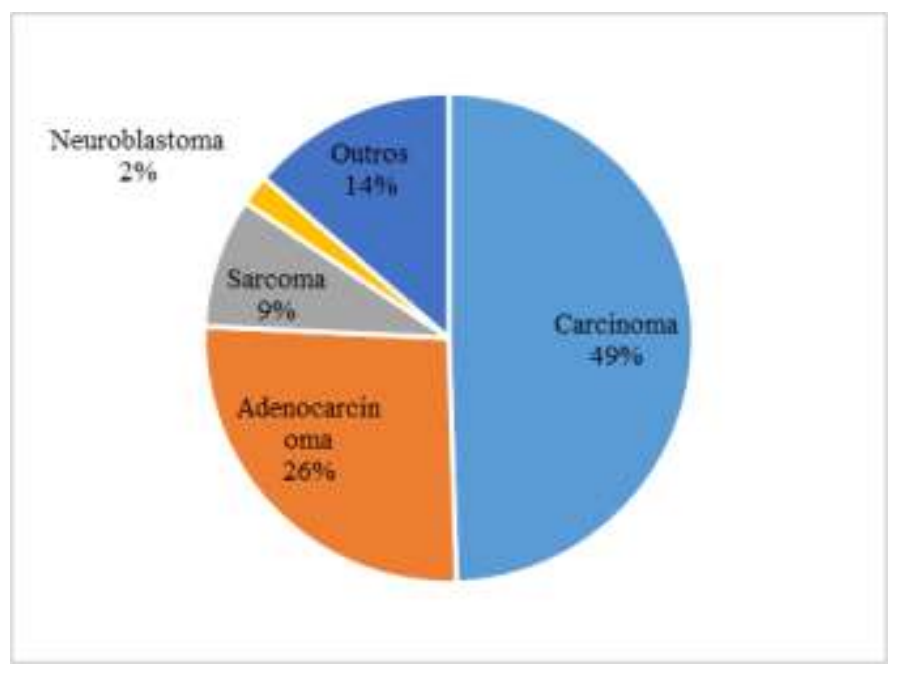

Fonte: Autores (2021).

\section{Conclusão}

A partir das trajetórias bibliográficas dos pesquisadores, esse estudo abordou sobre os locais do tumor primário que possuem metástase para a região bucomaxilofacial, bem como o local da metástase, gênero mais acometido e o tipo histológico mais comum.

O câncer da região bucomaxilofacial apresenta altas taxas de morbimortalidade. Assim, é necessário que o médico e/ou cirurgião dentista leve em consideração as metástases para essa região, pois, apesar de ser um caso raro, são sinais de neoplasias disseminadas, a fim de melhorar o prognóstico do paciente. Os sítios primários que mais metastatizam são pulmão, rim, mama e fígado. O gênero mais acometido é o masculino e a mandíbula, é o local mais acometido, seguido por gengiva e língua. O tipo histológico mais comum é o carcinoma.

Com isso, o levantamento bibliográfico sobre as metástases na região bucomaxilofacial se mostra importante, pois envolve estudos retrospectivos que possuem relação com o tema proposto e, ao mesmo, tempo, cria-se a possibilidade futura de novos pesquisadores abordarem o tema, criando novos trabalhos, para que haja melhora na detecção precoce do câncer de boca, melhorando seu prognóstico e nas taxas de sobrevida desses pacientes.

\section{Referências}

Andabak Rogulj, A., C. Tomasovic Loncaric, D. Muller, I. Blivajs, M. Andabak, V. Vucicevic Boras, \& Sekerija. M. (2018). “Solid Malignant Metastases in the Jaw Bones.” British Journal of Oral and Maxillofacial Surgery 56(8):705-8.

Cai, Z., Chao, Z., Lizhen, W., Ling, Z., Zhiyuan, Z., Hanguang, Z., \& Yan'na, W. (2016). “A Retrospective Study of Six Patients with Mandibular Metastatic Carcinoma." Oncology Letters 11(6):3650-54.

Canto, L. 2017. "Mariana Ferreira Fernandes Da Silva Incidência De Tumores Metastáticos Na Região Buco-Maxilo-Facial: Uma Meta-Análise.”

Chen, C. Y., Wen, C. W., Chih, H. T., Chang, W. S., \& Yuk, K. C. (2020). "Clinicopathological Study of 13 Cases of Intraoral Soft Tissue Metastatic Carcinomas." Journal of Dental Sciences 15(1):92-95.

Daley, T., \& Mark R. Darling. (2011). "Metastases to the Mouth and Jaws: A Contemporary Canadian Experience.” Journal of the Canadian Dental Association 77 .

Irani, S. (2016). "Metastasis to the Oral Soft Tissues: A Review of 412 Cases.” Journal of International Society of Preventive \& Community Dentistry 6(5):393-401.

Jham, B. C., Andrew R. S., Shawn A. McClure, \& Robert A. (2011). "Metastatic Tumors to the Oral Cavity: A Clinical Study of 18 Cases." Head and Neck Pathology 5(4):355-58.

Kaplan, I., Vadim R., Amir S., Benjamin S., Eli R., Adi G., Avraham H., Ran Y., Anna S-S, \& Marilena V. (2019). "Metastatic Tumors in Oral Mucosa and 
Research, Society and Development, v. 10, n. 12, e32101220076, 2021

(CC BY 4.0) | ISSN 2525-3409 | DOI: http://dx.doi.org/10.33448/rsd-v10i12.20076

Jawbones: Unusual Primary Origins and Unusual Oral Locations.” Acta Histochemica 121(8):151448.

Lawes, K. P., Martin D., \& Silvana Di P. (2013). "Delayed Metastasis to the Mandible of Esophageal Adenocarcinoma." Head and Neck Pathology 7(4):41620 .

Lee, Y. H., \& Jae Il L. (2017). "Metastatic Carcinoma of the Oral Region: An Analysis of 21 Cases." Medicina Oral, Patologia Oral y Cirugia Bucal 22(3):e359-65.

Liu, Y., Richard J. V., \& Elizabeth A. B. (2018). "Analytic Survey of 57 Cases of Oral Metastases.” Journal of Oral Pathology \& Medicine: Official Publication of the International Association of Oral Pathologists and the American Academy of Oral Pathology 47(3):275-80.

Maschino, F., J. Guillet, R. Curien, G. Dolivet, \& Bravetti, P. (2013). “Oral Metastasis: A Report of 23 Cases.” International Journal of Oral and Maxillofacial Surgery 42(2):164-68.

Metzger, K., Julius M., Dominik H., Maximilian P., Oliver R., Jürgen H., Kolja F., Michael E., \& Christian F. (2021). “Treatment Delay in Early-Stage Oral Squamous Cell Carcinoma and Its Relation to Survival.” Journal of Cranio-Maxillofacial Surgery 49(6):462-67.

Nawale, K. K., Monika V., Shubhada K., \& Asawari P. (2016). "Metastatic Tumors in the Jaw Bones: A Retrospective Clinicopathological Study of 12 Cases at Tertiary Cancer Center." Journal of Oral and Maxillofacial Pathology 20(2):252-55.

Owosho, A. A., Bin X., Arvin K., Sae H. K. Yom, J. R., Ronald A. Ghossein, J. M. H., \& Cherry L. E. (2016). "Metastatic Solid Tumors to the Jaw and Oral Soft Tissue: A Retrospective Clinical Analysis of 44 Patients from a Single Institution." Journal of Cranio-Maxillofacial Surgery 44(8):1047-53.

Reymond, N., Bárbara B. d'Á., and Anne J. R.. (2013). “Crossing the Endothelial Barrier during Metastasis.” Nature Reviews. Cancer 13(12):858-70.

Shimono, H., Hideaki H., Yu Oikawa, Y. M., Takeshi K., Hirofumi T., Kou K., Tohru I., \& Hiroyuki H. (2021). "Metastatic Tumors in the Oral Region: A Retrospective Chart Review of Clinical Characteristics and Prognosis.” Oral Surgery, Oral Medicine, Oral Pathology and Oral Radiology.

Shin, S. J., Jong L. R., Seung H C., Soon Y. N., Sang Y. K., Sung B. K., Sang W. L., \& Kyung J. C. (2012). "Metastatic Carcinomas to the Oral Cavity and Oropharynx." Korean Journal of Pathology 46(3):266-71.

Waltz, M. (2013). “Autism: A Social and Medical History.” Autism: A Social and Medical History 1-188.

Zalaudek, I., A. Ciarrocchi, S. Piana, G. Argenziano, F. Torricelli, V. Sancisi, G. Gandolfi, C. Longo, E. Moscarella, C. Banzi, \& Nicoli. (2015). “A Novel BRAF Mutation in Association with Primary Amelanotic Melanoma with Oral Metastases." Journal of the European Academy of Dermatology and Venereology : JEADV 29(2):387-90. 